\title{
Fungos anemófilos isolados de bibliotecas de instituições de ensino da Região Nordeste do Brasil
}

\section{Anemophilous fungi isolated from libraries of educational institutions in the Northeast of Brazil}

Davi Porfirio da Silva'(iD, Rodrigo José Nunes Calumby' (iD, Lais Nicolly Ribeiro da Silva'(D), Jayane Omena de Oliveira' (D), José Rafaelly Gaia de Sousa² (D), Delane Cristina da Silva² iD, Rossana Teotônio de Farias Moreira' (iD, Maria Anilda dos Santos Araújo3 (iD

' Universidade Federal de Alagoas, Maceió, Alagoas, Brasil

${ }^{2}$ Centro Universitário Cesmac, Maceió, Alagoas, Brasil

${ }^{3}$ Centro Universitário Tiradentes, Maceió, Alagoas, Brasil

\section{RESUMO}

INTRODUÇÃO: Estudos sobre micobiota anemófila de bibliotecas evidenciam ampla variedade de fungos, incluindo aqueles potencialmente patogênicos, oferecendo risco ocupacional e manifestações alérgicas a seus frequentadores. OBJETIVO: Avaliar a ocorrência de fungos anemófilos em bibliotecas de instituições de ensino da educação básica e superior da cidade de Maceió, estado de Alagoas, Brasil. MATERIAIS E MÉTODOS: Amostras do ambiente foram obtidas a partir da exposição de 55 placas de Petri contendo ágar Sabouraud com cloranfenicol em três bibliotecas de três instituições de ensino. As colônias fúngicas resultantes foram submetidas à identificação por meio da associação de aspectos macroscópicos e microscópicos, utilizando-se microcultivo. Ensaios fenotípicos complementares também foram utilizados. RESULTADOS: Das 55 amostras analisadas, foram obtidas 351 unidades formadoras de colônias (UFC), das quais 331 $(94,3 \%)$ corresponderam a fungos filamentosos e $20(5,7 \%)$ a leveduriformes. As espécies de fungos filamentosos mais frequentes foram Penicillium sp., Cladosporium sp., Alternaria sp., Aspergillus sp. e Curvularia sp., destacando-se maior predomínio de Penicillium sp. em uma biblioteca cujo ambiente não era climatizado, com 80 (22,7\%) UFC. CONCLUSÃO: Os resultados deste estudo evidenciam ampla variedade de fungos com potencial patogênico e toxigênico, que podem desencadear processos alérgicos, ratificando assim a importância do estabelecimento de protocolos de higiene e de desinfecção nesse tipo de ambiente.

Palavras-chave: Fungos; Contagem de Colônia Microbiana; Bibliotecas.

\begin{abstract}
INTRODUCTION: Studies on airborne mycobiota from libraries show a wide variety of fungi, including those potentially pathogenic, offering occupational risk and allergic manifestations to their visitors. OBJECTIVE: To evaluate the occurrence of anemophilous fungi in libraries of educational institutions of basic and higher education in the city of Maceió, Alagoas State, Brazil. MATERIALS AND METHODS: Samples of the air were obtained from the exposure of 55 Petri dishes containing Sabouraud agar with chloramphenicol in three libraries of three educational institutions. The resulting fungal colonies were subjected to identification through the association of macroscopic and microscopic aspects, using microculture. Complementary phenotypic assays were also performed. RESULTS: A total of 351 colony-forming units (CFU) were obtained from the 55 analyzed samples, of which 331 (94.3\%) were filamentous fungi and 20 (5.7\%) were yeasts. The most frequent filamentous fungus species were Penicillium sp., Cladosporium sp., Alternaria sp., Aspergillus sp., and Curvularia sp., with a greater predominance of Penicillium sp. in a library that was not acclimatized, with 80 (22.7\%) CFU. CONCLUSION: The results of this study show a great diversity of fungi with pathogenic and toxigenic potential, which can trigger allergic processes, thus confirming the importance of establishing hygiene and disinfection protocols in this type of environment.
\end{abstract}

Keywords: Fungi; Microbial Colony Count; Libraries. 


\section{INTRODUÇÃO}

Fungos são organismos eucarióticos, heterotróficos, unicelulares ou pluricelulares, amplamente distribuídos na natureza, encontrados no ar atmosférico, no solo, na água, nos vegetais, em detritos em geral, nos alimentos, no homem e em outros animais ${ }^{1,2,3,4}$. A disseminação desses microrganismos pode ocorrer sob várias formas. Quando essa propagação se dá pelo ar, são denominados de fungos anemófilos ou fungos alergizantes, cujos esporos são aeroalérgenos, responsáveis por manifestações respiratórias, que podem assumir caráter oportunista em hospedeiros imunocomprometidos ou submetidos à alta exposição ${ }^{1,4}$.

A concentração de fungos anemófilos no ambiente depende de vários fatores, dentre esses se destacam o tipo de climatização de ambientes fechados, intensidade de atividade humana e outros aspectos ambientais, tais como correnteza de ar, precipitação pluviométrica, pressão barométrica, nebulosidade, temperatura, umidade relativa do ar e incidência de raios solares ${ }^{2,5}$. Em bibliotecas, o ambiente torna-se propício à propagação de microrganismos, especialmente fungos anemófilos, devido as suas condições especiais de ventilação/climatização, umidade e temperatura ${ }^{3,6}$.

Estudos sobre a micobiota anemófila de bibliotecas mostram uma ampla variedade de fungos, incluindo aqueles potencialmente patogênicos, cuja presença representa um risco ocupacional e pode desencadear manifestações alérgicas em seus frequentadores $3,4,6$. Sabe-se que indivíduos confinados em ambientes fechados, com ventilação e climatização artificiais, a exemplo de salas de aula, bibliotecas, teatros, cinemas e outros, podem apresentar sintomas persistentes, que incluem dores de cabeça, prurido, irritação e edema ocular e das mucosas, desconforto na orofaringe e mal-estar geral ${ }^{4}$, comprometendo a qualidade de vida desses.

Devido a esses fatos, boas práticas de limpeza e higienização ambiental são implementadas em muitas bibliotecas por meio de medidas simples, como o uso de pincéis, flanelas (secas) ou até mesmo aspirador de pó $^{3}$. $\bigcirc$ ar desses ambientes, sobretudo ao se considerar as características dos fungos anemófilos, é mais afetado, podendo apresentar maior número de unidades formadoras de colônias (UFC) quando comparado com superfícies e livros ${ }^{6}$. Entretanto, apesar da existência de normas de higienização ambiental, há poucos estudos e iniciativas de atenção à qualidade do ar dessas unidades ${ }^{4,7}$.

Nesse contexto, há a necessidade de consolidação de informações sobre os fungos anemófilos, o aumento de estudos sobre microrganismos com potencial alergênicos e a busca de indicadores ambientais, como a presença desses fungos. No caso de bibliotecas, em particular, o interesse surge das implicações para a conservação de livros e quanto à condição de saúde de trabalhadores e frequentadores, considerando que os problemas de saúde mais reportados por trabalhadores desse serviço estão relacionados a mecanismos alérgicos ${ }^{8,9}$. Portanto, este estudo teve como objetivo avaliar a ocorrência de fungos anemófilos em bibliotecas de instituições de ensino de nível básico e superior da cidade de Maceió, estado de Alagoas, Brasil.

\section{MATERIAIS E MÉTODOS}

\section{CARACTERIZAÇÃO DO AMBIENTE E COLETA DAS AMOSTRAS}

As amostras foram colhidas em bibliotecas de duas instituições de ensino superior, sendo uma pública e uma privada (espaços A e C), ocupando uma área geográfica de $1.560 \mathrm{~m}^{2}$ e $730 \mathrm{~m}^{2}$, respectivamente, e em uma biblioteca de um complexo de escolas públicas de nível fundamental e médio (espaço B), com área de $850 \mathrm{~m}^{2}$, localizadas em Maceió. Os ambientes das instituições de nível superior possuíam condicionadores de ar de 12.000 BTU, enquanto na instituição de nível médio e fundamental havia somente ventiladores de teto.

Conforme a observação dos pesquisadores, as três bibliotecas apresentavam iluminação artificial, sem incidência de luz solar, e a higienização dos pisos ocorria diariamente, utilizando saneantes domissanitários. Além disso, com o auxílio de um pano úmido, era feita a remoção diária de poeira de balcões de madeira, mesas, cadeiras, armários, arquivos, carrinhos de transporte de livros, persianas, caixilhos das janelas e computadores. A limpeza das capas dos livros era realizada trimestralmente no espaço $C$ e semestralmente nos espaços $A$ e $B$, utilizando algodão umedecido em álcool ou acetato de etila. As estantes eram higienizadas com pano levemente umedecido em álcool, tendo-se o cuidado para não molhar os livros. No espaço C, além da limpeza descrita anteriormente, a remoção do pó era feita por aspirador a cada dois meses.

No momento das coletas, foram realizadas medições de temperatura e umidade relativa do ar de cada ambiente com termo-higrômetro digital. Para a coleta de fungos anemófilos, foi utilizada a técnica de sedimentação de esporos, que consiste na exposição de placas de Petri contendo meio de cultivo a altura de 1,2 $\mathrm{m}$ a partir do solo por $30 \mathrm{~min}^{10}$. Desse modo, foram realizadas exposições de 55 placas de Petri de $90 \mathrm{~mm}$ contendo ágar Sabouraud dextrose (ASD), acrescido de cloranfenicol na concentração de 50 mg/L, em locais estratégicos de acesso constante, como mesas, estantes e balcões, levando-se em consideração a dimensão do ambiente e uma equidistância entre uma placa e outra de 8,5 m. Respeitando essa equidistância, 15 placas foram expostas em cada um dos espaços B e C, e 25 placas no espaço $A$. No espaço $A$, de $1.560 \mathrm{~m}^{2}$, considerou-se dimensões iguais de 39,5 x 39,5 m, cuja distância no eixo $X$ foi de 8,75 m e em $Y$ de 9,7 m, o que padronizou a equidistância de $8,5 \mathrm{~m}$ entre as placas, de forma a respeitar o tráfego de usuários e funcionários. $\bigcirc$ mesmo raciocínio foi empregado para os espaços $B$ e C, com área de $850 \mathrm{~m}^{2}$ e $730 \mathrm{~m}^{2}$, respectivamente, com dimensões de 29,1 m x 29,1 m e de $27 \mathrm{~m} \times 27 \mathrm{~m}$, para a distribuição das 15 placas. Transcorrido o tempo de exposição, as placas foram devidamente etiquetadas, acondicionadas e transportadas ao Laboratório de 
Micologia do Centro Universitário Cesmac, onde foram incubadas à temperatura ambiente $\left(28 \pm 2^{\circ} \mathrm{C}\right)$ e observadas diariamente por um período de sete dias.

\section{ISOLAMENTO E IDENTIFICAÇÃO DOS FUNGOS}

Após constatação de crescimento fúngico nas placas, foi realizada a análise quantitativa por meio da contagem do número de UFC, diferenciando-as em leveduriformes e filamentosas.

Para o isolamento dos fungos, inicialmente, foi realizada a purificação por meio da transferência de fragmentos de colônias para o preparo de uma suspensão em água destilada esterilizada e, em seguida, a semeadura por esgotamento em placas de Petri contendo ASD adicionado de cloranfenicol $(50 \mathrm{mg} / \mathrm{L}$ ). As placas foram mantidas à temperatura ambiente $\left(28 \pm 2^{\circ} \mathrm{C}\right)$ para o desenvolvimento de colônias isoladas. Constatado o crescimento, essas colônias foram transferidas para tubos de ensaio contendo ágar batata dextrose para manutenção e posterior identificação ${ }^{11,12,13}$.

A identificação dos fungos filamentosos baseou-se na associação das características macro e micromorfológicas da cultura. As características macroscópicas, como textura, formato e coloração no verso e reverso da colônia, juntamente com as características microscópicas, tais como estruturas reprodutivas e outras, como clamidósporos, hifas (presença ou ausência de septos), coloração das hifas e dos esporos (hialinos ou demáceos), foram comparadas aos critérios adotados por Hoog et al. ${ }^{11}$, Lacaz et al. ${ }^{12}$, Sidrim e Rocha ${ }^{13}$, Zaitz et al. ${ }^{14}$ e Mezzari e Fuentefria ${ }^{15}$. Para melhor identificação e visualização das estruturas dos fungos filamentosos, foi feito o microcultivo em lâmina, pela técnica descrita por Riddell'b, utilizando ágar Lactrimel para estimular a esporulação.
Para identificação dos fungos leveduriformes, foram realizadas as análises macromorfológica das colônias e microscópica pela técnica de microcultivo em ágar fubá. $\bigcirc$ resultado foi associado ao teste de tubo germinativo, formação de clamidósporos em ágar Tween 80 (Difco) e ao padrão de assimilação e fermentação de diversas fontes de carbono e nitrogênio ${ }^{14}$.

\section{RESULTADOS}

No momento da exposição das placas, foram observados registros térmicos de $24,6{ }^{\circ} \mathrm{C}, 28,1^{\circ} \mathrm{C}$ e $21,6{ }^{\circ} \mathrm{C}$ e umidade de $68 \%, 69 \%$ e $59 \%$ nos acervos $\mathrm{A}$, $B$ e $C$, respectivamente, que originaram 55 amostras no total (Tabela 1).

Nas 55 amostras analisadas, foram obtidas 351 UFC, das quais 331 UFC (94,3\%) eram fungos filamentosos e 20 UFC $(5,7 \%)$ fungos leveduriformes. A contagem global de colônias nos acervos bibliográficos A, B e C resultou em 212 (60,4\%), 123 (35,0\%) e 16 $(4,6 \%)$ UFC, respectivamente, conforme tabela 2 .

A biblioteca A apresentou maior contagem de UFC, tanto de fungos filamentosos quanto de leveduriformes. Em relação à identificação dos fungos, observou-se uma maior ocorrência de Penicillium sp. (1 15 UFC), seguido por Cladosporium sp. (79 UFC), Alternaria sp. (20 UFC), Aspergillus sp. (20 UFC) e Curvularia sp. (18 UFC). A maior diversidade fúngica foi verificada na biblioteca $A$, registrando 15 gêneros, sendo Cladosporium sp. (69 UFC) o fungo predominante, seguido por Penicillium sp. (32 UFC) e Curvularia sp. (16 UFC). Na biblioteca B, onde não havia climatização, o gênero de maior ocorrência foi Penicillium sp. (80 UFC), enquanto na biblioteca C predominou o gênero Aspergillus sp. (6 UFC). Não foram identificados 13,9\% (49 UFC) dos fungos devido à ausência de estruturas reprodutivas (Tabela 3).

Tabela 1 - Registro de temperatura e umidade do ambiente, no momento de exposição das placas, e número de amostras obtidas nas bibliotecas A, B e C da cidade de Maceió, estado de Alagoas, Brasil

\begin{tabular}{ccccc}
\hline \multirow{2}{*}{ Biblioteca } & \multicolumn{2}{c}{ Parâmetros termo-higrométricos } & Número de amostras & $\%$ \\
& Temperatura ${ }^{\circ} \mathrm{C}$ & URA & 25 & 45,4 \\
A & 24,6 & 68 & 15 & 27,3 \\
B & 28,1 & 59 & 15 & 27,3 \\
C & 21,6 & 69 & 55 & 100,0 \\
\hline Total & & &
\end{tabular}

URA: Umidade relativa do ar.

Tabela 2 - Ocorrência de UFC de fungos filamentosos e leveduriformes nas bibliotecas A, B e C da cidade de Maceió, estado de Alagoas, Brasil

\begin{tabular}{cccccrrr}
\hline \multirow{2}{*}{ Bibliotecas } & \multicolumn{2}{c}{ Filamentosos } & \multicolumn{2}{c}{ Fungos } & \multicolumn{2}{c}{ Leveduriformes } & Total \\
& FA (UFC) & FR (\%) & FA (UFC) & FR (\%) & & \\
\hline A & 198 & 56,4 & 14 & 4,0 & 212 & 60,4 \\
B & 118 & 33,6 & 5 & 1,4 & 123 & 35,0 \\
C & 15 & 4,3 & 1 & 0,3 & 16 & 4,6 \\
\hline Total & 331 & 94,3 & 20 & 5,7 & 351 & 100,0 \\
\hline
\end{tabular}

FA: Frequência absoluta; FR: Frequência relativa. 
Tabela 3 - Microbiota fúngica anemófila identificada nas bibliotecas A, B e C da cidade de Maceió, estado de Alagoas, Brasil

\begin{tabular}{|c|c|c|c|c|c|c|c|c|}
\hline \multirow{3}{*}{$\begin{array}{l}\text { Gêneros/ } \\
\text { Espécies }\end{array}$} & \multicolumn{6}{|c|}{ Bibliotecas } & \multirow{3}{*}{ Total } & \multirow{3}{*}{$\%$} \\
\hline & \multicolumn{2}{|c|}{$A$} & \multicolumn{2}{|c|}{ B } & \multicolumn{2}{|c|}{ C } & & \\
\hline & FA (UFC) & FR (\%) & FA (UFC) & FR (\%) & FA (UFC) & FR (\%) & & \\
\hline Acremonium sp. & 2 & 0,6 & - & - & 2 & 0,6 & 4 & 1,2 \\
\hline Alternaria sp. & 15 & 4,3 & 5 & 1,4 & - & - & 20 & 5,7 \\
\hline Aspergillus sp. & 14 & 4,0 & - & - & 6 & 1,7 & 20 & 5,7 \\
\hline Bipolaris sp. & 2 & 0,6 & - & - & - & - & 2 & 0,6 \\
\hline Cladosporium sp. & 69 & 19,6 & 8 & 2,3 & 2 & 0,6 & 79 & 22,5 \\
\hline Curvularia sp. & 16 & 4,6 & 2 & 0,6 & - & - & 18 & 5,2 \\
\hline Fonsecaea sp. & 5 & 1,4 & - & - & - & - & 5 & 1,4 \\
\hline Fusarium sp. & 10 & 2,8 & - & - & - & - & 10 & 2,8 \\
\hline Mycelia sterilia & - & - & 1 & 0,3 & - & - & 1 & 0,3 \\
\hline Neurospora sp. & 1 & 0,3 & - & - & - & - & 1 & 0,3 \\
\hline Nigrospora sp. & - & - & 1 & 0,3 & - & - & 1 & 0,3 \\
\hline Penicillium sp. & 32 & 9,1 & 80 & 22,7 & 3 & 0,8 & 115 & 32,6 \\
\hline Rhodotorula sp. & 5 & 1,4 & 1 & 0,3 & 1 & 0,3 & 7 & 2,0 \\
\hline Scopulariopsis sp. & 3 & 0,9 & - & - & - & - & 3 & 0,9 \\
\hline Trichosporon sp. & 7 & 2,0 & 2 & 0,6 & - & - & 9 & 2,6 \\
\hline Ulocladium sp. & 4 & 1,1 & - & - & - & - & 4 & 1,1 \\
\hline Veronaea sp. & 2 & 0,6 & 1 & 0,3 & - & - & 3 & 0,9 \\
\hline Não identificados & 25 & 7,1 & 22 & 6,2 & 2 & 0,6 & 49 & 13,9 \\
\hline Total & 212 & 60,4 & 123 & 35,0 & 16 & 4,6 & 351 & 100,0 \\
\hline
\end{tabular}

UFC: Unidade formadora de colônia; FA: Frequência absoluta; FR: Frequência relativa. Sinal convencional utilizado: - Dado numérico igual a zero, não resultante de arredondamento.

Diversas culturas fúngicas foram obtidas após a coleta das amostras por sedimentação espontânea nas três bibliotecas estudadas. Na figura 1, estão apresentados os aspectos macroscópicos e microscópicos dos seis fungos mais prevalentes e que exibiram mais de 10 UFC nos ambientes avaliados, sendo esses representados por Alternaria sp., Aspergillus sp., Cladosporium sp., Curvularia sp., Fusarium sp. e Penicillium sp.

As limitações deste estudo estão relacionadas à quantidade de espaços estudados e à falta de estrutura para realizar a identificação molecular desses fungos.

\section{DISCUSSÃO}

Os resultados obtidos neste estudo assemelham-se aos relatados por Menezes et al. ${ }^{17}$, no que se refere à diversidade fúngica encontrada, já que obtiveram 220 UFC a partir de 50 exposições em uma biblioteca climatizada de uma instituição pública de ensino superior no nordeste do Brasil. Ambientes fechados climatizados tendem ao acúmulo de células fúngicas dispersas no ar, como evidenciado por Ribeiro e Lubisco ${ }^{6}$, ao compararem a concentração de UFC de ambiente interno com 0 externo de uma biblioteca, onde $\circ$ primeiro apresentou aproximadamente $\circ$ dobro de concentração de UFC que o segundo.

As bibliotecas são espaços que propiciam condições ideais para o crescimento de microrganismos, uma vez que concentram grande quantidade de matéria orgânica, incluindo papel, cola de amido, couro e tecidos ${ }^{18}$. Por isso, evidências apontam que, mesmo em condições controladas, há contaminação por fungos em superfícies e no ar desses ambientes ${ }^{19}$.

No espaço C, foi observado um menor número de UFC e espécies de fungos filamentosos quando comparado à biblioteca $A$, que também é climatizada. Esse fato pode estar relacionado à sistematização da limpeza desse local e menor temperatura e umidade verificada no momento da coleta. A biblioteca C adotava práticas regulares de limpeza e higienização; além disso, o ambiente apresentava melhor refrigeração, especialmente por possuir menor fluxo de pessoas no local, observação feita durante o período do estudo, quando comparado à biblioteca $\mathrm{A}$, que oferecia 88 cursos presenciais, e a C, 13 cursos e ser constituída por uma área menor. Assim, a maior frequência de fungos no espaço $A$ pode estar relacionada à maior visitação nesse espaço por alunos e funcionários, visto que essa biblioteca serve de referência para outras instituições de ensino e pesquisa do estado. Ademais, é importante considerar que os equipamentos de climatização são frequentemente desligados no período noturno e aos fins de semana nessa biblioteca, o que causa uma variabilidade de temperatura, sobretudo em decorrência das amplitudes térmicas experimentadas na Região Nordeste. 

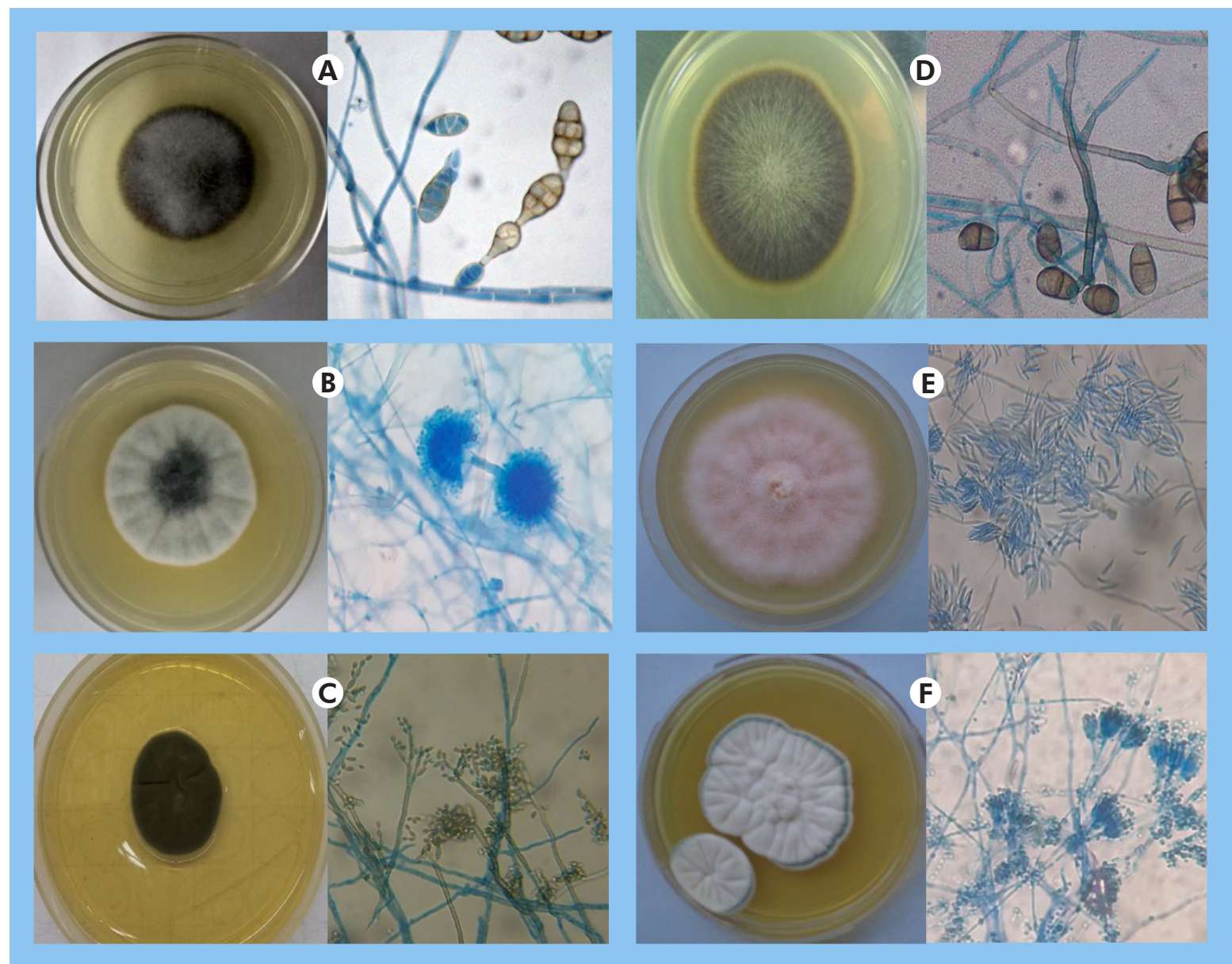

A: Alternaria sp.; B: Aspergillus sp.; C: Cladosporium sp.; D: Curvularia sp.; E: Fusarium sp.; F: Penicillium sp. Lâminas coradas com lactofenol azul-algodão.

Figura 1 - Aspectos macroscópicos e microscópicos dos fungos que apresentaram maior prevalência no ambiente das bibliotecas A, B e C, cultivados em placas de Petri contendo ágar batata dextrose a $28{ }^{\circ} \mathrm{C}$, na cidade de Maceió, estado de Alagoas, Brasil

O espaço $B$, segundo em número de UFC e diversidade fúngica, não possuía equipamentos de climatização, sendo observada a maior temperatura registrada durante a coleta. Além disso, as baixas taxas de circulação de ar em ambientes internos podem ocasionar um aumento considerável na concentração de polventes biológicos do $a^{20}$. Pantoja et al. ${ }^{21}$ esclarecem que 0 crescimento de fungos ocorre de maneira mais rápida quando a umidade relativa do ar está acima da taxa de 65\%, também evidenciado nos ambientes $\mathrm{A} e$ B. Segundo Pereira e Lemos 22 , a temperatura exerce uma função primordial tanto sobre o crescimento, germinação e esporulação dos fungos como também sobre o seu metabolismo.

Fatores como higienização, temperatura e luminosidade estão diretamente relacionados à maior diversidade fúngica ${ }^{20}$. A limpeza inadequada, com uso de métodos que facilitam a dispersão aérea de microrganismos, por exemplo, pode contribuir para esse fato, como ocorre com o uso de vassouras. É importante considerar que a diversidade fúngica relatada neste estudo pode estar relacionada às ineficientes condições de higienização e climatização, que variam em cada espaço estudado ${ }^{21}$, sendo observada maior prevalência de fungos nas bibliotecas de instituições públicas.
A fim de evitar ou diminuir a proliferação de fungos e outros microrganismos em bibliotecas, é recomendada a prática da limpeza regular, proporcionando a conservação do acervo, a manutenção da qualidade do ar e, consequentemente, preservando a saúde dos seus frequentadores. A higienização corresponde principalmente à retirada de material particulado (poeira, pólen, escamas de pele humana, pelos, entre outros) dos documentos e superfícies do ambiente, devendo ser realizada periodicamente, com o uso de técnicas apropriadas ${ }^{6}$. $\bigcirc$ espaço físico das bibliotecas deve ser suficientemente arejado, iluminado e apresentar valores de temperatura (entre 20 e $23^{\circ} \mathrm{C}$ ) e umidade ( 55 a $65 \%$ ) adequados à preservação de livros e documentos ${ }^{21}$. Além disso, torna-se essencial a higienização mensal dos filtros dos condicionadores de ar nos acervos que dispõem de climatização, a fim de diminuir o crescimento de microrganismos, uma vez que o acúmulo de umidade e material orgânico nesses equipamentos pode torná-los poderosas fontes dispersoras de patógenos ${ }^{23,24}$.

No que concerne à variabilidade de espécies fúngicas, estudos publicados corroboram com os resultados obtidos nesta pesquisa, pois as coletas realizadas ao longo dos anos em espaços internos de bibliotecas mostraram que, entre os fungos que 
contaminam esses espaços, predominam espécies do gênero Aspergillus e Penicillium ${ }^{8,17,20}$. No presente estudo, as espécies de fungos filamentosos mais frequentes foram Penicillium sp. e Cladosporium sp.; entretanto, Alternaria sp., Aspergillus sp. e Curvularia $\mathrm{sp}$. também apresentaram frequência superior às outras espécies identificadas.

Estudos ambientais realizados nos últimos 20 anos com ar dos ambientes de bibliotecas evidenciaram que espécies de Cladosporium, Aspergillus e Penicillium são quase onipresentes e podem produzir numerosos conídios que são facilmente dispersados pelo $\mathrm{ar}^{25}$. Segundo Martins $^{26}$, a diversidade de fungos presente em ambientes fechados é resultante da disseminação ocasionada pelas correntes de ar, entre outros fatores bióticos do ambiente externo, e está relacionada especialmente ao aparecimento de gêneros característicos da microbiota local de determinada região.

Nesse sentido, espécies pertencentes aos gêneros Aspergillus, Penicillium e Verticillium apresentam atividade celulolítica, sendo capazes de deteriorar materiais ricos em celulose, tais como madeira, livros e quadros ${ }^{27}$. Além do mais, os gêneros de fungos que são encontrados com maior frequência no ar são Aspergillus sp., Penicillium sp., Fusarium sp., Cladosporium sp., Curvularia sp., Neurospora sp. e Alternaria sp., sendo Aspergillus e Penicillium considerados os colonizadores primários de superfícies e interiores ${ }^{28}$.

Em relação aos fungos filamentosos identificados neste estudo, grande parte desses microrganismos está comumente associada a processos patológicos. Fungos anemófilos, quando inalados, podem desencadear doenças que vão desde manifestações alérgicas respiratórias, tais como asma e rinite, até infecções graves em indivíduos imunocomprometidos ${ }^{20}$.

Entre essas espécies, fungos do gênero Aspergillus causam maior preocupação, por poder ocasionar desde reações alérgicas até aspergilose, doença caracterizada por um quadro clínico broncopulmonar e que pode se disseminar, comprometendo diversos órgãos ${ }^{20}$. A aspergilose invasiva está relacionada a uma diversidade de cenários clínicos, comumente variável em relação às manifestações, apresentando uma taxa de mortalidade muito elevada 29 .

Embora as espécies de Penicillium sejam saprófitas, ubíquas do solo e, consequentemente, sobrevivam de substâncias orgânicas biodegradáveis e prefiram climas frios a moderados, sua colonização está relacionada à capacidade de crescimento em uma variedade de temperaturas, habilidade de sobreviver em diferentes faixas de atividade de água e condições físico-químicas, as quais são encontradas em bibliotecas, tornando esse ambiente propício para a sua dispersão ${ }^{30}$.

Além disso, Cladosporium tem sido o gênero fúngico mais comum em diversos estudos de ambientes internos climatizados, sendo considerado um fungo dominante universal31,32,33,34,35,36. Muitas espécies de Cladosporium são reconhecidas como patógenos emergentes, responsáveis por infecções pulmonares, cutâneas e problemas relacionados ao sistema respiratório, frequentemente associados a queixas asmáticas ${ }^{37,38}$.
Em menor frequência, foi observada a presença de fungos leveduriformes nos ambientes de estudo. Embora tenha sido relatado o isolamento desses fungos, pouca atenção é dada aos mesmos, quando comparados aos fungos filamentosos responsáveis por desencadear processos alérgicos de forma iminente, a exemplo do Aspergillus, enquanto fungos leveduriformes não produzem reações alérgicas ou respiratórias de imediato, embora possam ser danosos a indivíduos com imunocomprometimento ${ }^{8}$. É importante ressaltar que podem estar relacionados a infecções superficiais e sistêmicas, especialmente naqueles indivíduos com comprometimento do sistema imunológico ${ }^{39}$.

Entre as espécies leveduriformes isoladas, Trichosporon sp. apresentou maior frequência, com 9 UFC (2,6\%). Trichosporon é um fungo que pode fazer parte da microbiota normal da pele humana e trato gastrointestinal, e 17 espécies desse gênero são clinicamente relevantes ${ }^{40,41,42}$ por serem causadoras de piedra branca, pneumonite por hipersensibilidade e vários tipos de infecções invasivas, principalmente em indivíduos imunocomprometidos ${ }^{41}$, sendo, portanto, considerado oportunista.

Outra levedura identificada foi Rhodotorula sp., considerada comensal e que está presente nas vias respiratórias, microbiota gastrointestinal e genital humana e disseminada no meio ambiente. Pode causar infecções oportunistas em pacientes imunocomprometidos, as quais variam de endocardite, meningite, ventriculite e peritonite, até casos de fungemia potencialmente fatais ${ }^{43,44,45}$.

Os resultados deste estudo demonstram a necessidade da melhoria das medidas de desinfecção ambiental para manutenção do acevo de bibliotecas e prevenção de doenças ocasionadas por fungos em seus frequentadores.

\section{CONCLUSÃO}

Os resultados deste estudo evidenciam a importância de rotinas de desinfecção ambiental em espaços fechados, como as bibliotecas, para a prevenção de doenças em seus frequentadores e a conservação do acervo, visto que o risco à saúde pode ser maior ou menor, dependendo das medidas de controle ambiental adotadas, da frequência da higienização do acervo e do ambiente em geral, do monitoramento da temperatura e da umidade relativa do ar.

\section{CONFLITOS DE INTERESSES}

Os autores declaram não haver conflitos de interesses em relação à pesquisa.

\section{CONTRIBUIÇÃO DOS AUTORES}

José Rafaelly Gaia de Sousa, Delane Cristina da Silva e Maria Anilda dos Santos Araújo foram responsáveis pela coleta, processamento e identificação das amostras. Davi Porfirio da Silva, Rodrigo José Nunes Calumby, Lais Nicolly Ribeiro da Silva, Jayane Omena de Oliveira e Rossana Teotônio de Farias Moreira foram responsáveis pela elaboração e aprovação do manuscrito. 


\section{REFERÊNCIAS}

1 Trabulsi LR, Flávio A. Microbiologia. 4. ed. São Paulo: Atheneu; 2004.

2 Rêgo CM, Santos FS. Ocorrência de fungos anemófilos e sua relação com fatores abióticos em Barreiras, Bahia. Rev Bras Bioci. 2015 out-dez;13(4):265-71.

3 Calegari GM, Novais VP, Bianco ME, Gois RV, Sobral FOS, Marson RF. Ocorrência de fungos em superfícies de livros e avaliação da eficácia do ozônio na desinfecção de uma biblioteca do norte do país. Braz J Surg Clin Res. 2017 dez-2018 fev;21 (3):27-31.

4 Lima MLF, Lima JS, Silva MT. Fungos anemófilos: avaliação da microbiota do ar em ambientes interno e externo. Essentia (Sobral). $2019 ; 20(1): 88-95$.

5 Souza PMS, Andrade SL, Lima AF. Pesquisa, isolamento e identificação de fungos anemófilos em restaurantes self-service do centro de Maceió/AL. Cad Grad Cienc Biol Saude. 2013 nov; 1 (3): 1 47-54.

6 Ribeiro ALPC, Lubisco NML. Redução de fungos em ambiente de biblioteca: viabilidade de aplicação de neblina ativada. Perspect Gest Conhec. 2016 jul-dez;6(2):250-60.

7 Portela PO, Kozusny-Andreani DI. Caracterização microbiológica em ambiente específico de uma biblioteca universitária em sua composição e qualidade. Em Questao. 2019 set-dez;25(3):373-89.

8 Pinheiro AC, Viegas C, Veríssimo C, Brandão J, Macedo MF. Bibliotecas na saúde... e a saúde nas bibliotecas? In: 12a Jornadas APDIS; 2016 abr 20-22; Coimbra, PT. Coimbra: Universidade de Coimbra; 2016.

9 Braga RS, Azevedo AK, Carneiro LF, Legey ALC, Motter AA. Prevalence of respiratory symptoms in library workers in a public university. Rev Saude Publica Parana. 2018 Jul;1(1):74-82.

10 Gambale W, Croce J, Costa-Manso E, Croce M, Sales M. Library fungi at the University of São Paulo and their relationship with respiratory allergy. J Investig Allergol Clin Immunol. 1993 Jan-Feb;3(1):45-50.

11 Hoog GS, Guarro J, Gené J, Figueras MJ. Atlas of clinical fungi. 2th ed. Spaim: CBS; 2000.

12 Lacaz CS, Porto E, Martins JEC, Heins-Vaccari EM, Melo NT. Micologia médica. 9. ed. São Paulo: Sarvier; 2002.
13 Sidrim JJC, Rocha MFG. Micologia médica à luz de autores contemporâneos. 2. ed. Rio de Janeiro: Guanabara Koogan; 2010.

14 Zaitz C, Campbell I, Marques SA, Ruiz LRB, Framil VMS. Compêndio de micologia médica. 2. ed. São Paulo: Guanabara Koogan; 2010.

15 Mezzari A, Fuentefria AM. Micologia no laboratório clínico. Rio de Janeiro: Manole; 2012.

16 Riddell RW. Permanent stained mycological preparations obtained by slide culture. Mycologia. 1950 Mar-Apr;42(2):265-70.

17 Menezes EA, Alcanfor AC, Cunha FA. Fungos anemófilos na sala de periódicos da biblioteca de ciências da saúde da Universidade Federal do Ceará. RBAC. 2006;38(3):155-8.

18 Campos FM, Golin R, Caixeta FC, Sanches L, Caixeta DS. Avaliação quanti-qualitativa do ar interior de uma biblioteca pública do município de Cuiabá-MT. Eng Sci. 2017;1(6):95-105.

19 Bortoletto ME, Machado RR, Coutinho E. Contaminação fúngica do acervo da biblioteca de manguinhos da Fundação Oswaldo Cruz: ações desenvolvidas para sua solução. Enc Bibli: R Eletr Bibliotecon Ci Inf. 2002;7(14)9-18.

20 Rosa H, Lemos JA, Costa CR, Silva MRR, Fernandes OFL. Ocorrência de fungos filamentosos em acervo da Faculdade de Medicina da Universidade Federal de Goiás. Rev Patol Trop. 2008 jan-abr;37(1)65-9.

21 Pantoja LDM, Rizzo RS, Carvalho BS, Ferreira VC, Galas KS, Fonseca FRM, et al. Constituição da micobiota aérea de bibliotecas públicas no município de fortaleza, estado do Ceará, Brasil. Enc Bibli: R Eletr Bibliotecon Ci Inf. 2012;17(34): $31-41$.

22 Pereira LTC, Lemos JLS. Os fungos filamentosos, uma opção em estudo para a biorremediação II. In: $11^{a}$ Jornada de Iniciação Científica; 2003; Rio de Janeiro, RJ. Rio de Janeiro: CETEM/MCT; 2003.

23 Honorato GM. Verificação de fungos anemófilos na U.T.I do Hospital Santa Lucinda (Sorocaba/SP), antes e depois de sua limpeza. Rev Eletronica Biol. 2009;2(3):19-31.

24 Mota RJBS, Gil RGB, Lima FB, Moraes FAB, Farias AS. Qualidade do ar interno no ambiente hospitalar: uma revisão integrativa. Rev Saude. $2014 ; 8(1 / 2): 44-52$.

25 Pinheiro AC, Sequeira SO, Macedo MF. Fungi in archives, libraries, and museums: a review on paper conservation and human health. Crit Rev Microbiol. 2019 Sep-Nov;45(5-6):686-700. 
26 Martins OA. Fungos anemófilos e leveduras isolados em ambientes de laboratórios de microbiologia em instituição de Ensino Superior [Dissertação]. Pelotas (RS): Universidade Federal de Pelotas, Faculdade de Veterinária; 2016.

27 Monteiro MCP. Identificação de fungos dos gêneros Aspergillus e Penicillium em solos preservados no cerrado [Dissertação]. Lavras (MG): Universidade Federal de Lavras; 2012.

28 Sobral LV. Fungos anemófilos em ambientes climatizados: prevalência, produção de enzimas e atividade antibacteriana [Dissertação]. Vitória de Santo Antão (PE): Universidade Federal de Pernambuco; 2016.50 p.

29 Carvalho LIC. Aspergillus e aspergilose: desafios no combate da doença [Dissertação]. Porto (PT): Universidade Fernando Pessoa; 2013.

30 Singh S, Khajuria R. Penicillium enzymes for the textile industry. In: Gupta VK, Rodriguez-Couto S, editors. New and future developments in microbial biotechnology and bioengineering. Florida: Elsevier; 2018. Chapter 11; p. 201-15.

31 Ribeiro EL. Fungos na biodeterioração de livros em ambientes bibliotecários nos últimos 35 anos (1977 - 2012). Rev Bras Bibliot Doc. 2013 jan-dez;9(1):17-27.

32 Grava S, Lopes FAD, Cavallazzi RS, Grassi MFNN, Svidzinski TIE. Um caso raro de pneumonia hemorrágica por Cladosporium cladosporioides. J Bras Pneumol. 2016 set-out;42(5):392-4.

33 Calumby RJN, Silva JA, Silva DP, Moreira RTF, Araújo MAS, Almeida LM, et al. Isolamento e identificação da microbiota fúngica anemófila em Unidade de Terapia Intensiva. Braz J Develop. 2019 out;5(10):19708-22.

34 Gonçalves CL, Mota FV, Ferreira GF, Mendes JF, Pereira EC, Freitas $\mathrm{CH}$, et al. Airborne fungi in an intensive care unit. Braz J Biol. 2018 May-Aug;78(2):265-70.

35 Schoenlein-Crusius IH, Trufem SFB, Grandi RAP, Milanez Al, Pires-Zottarelli CLA. Airborne fungi in the region of Cubatão, São Paulo State, Brazil. Braz J Microbiol. 2001 Jan-Mar;32(1):61-5.
36 Souza AKP, Nascimento JPM, Araújo MAS, Pedrosa KPS, Tenorio BM, Pires LLS, et al. Airborne fungi in neonatal intensive care unit of a public hospital in Brazil. Int J Curr Microbiol App Sci. 2019;8(12):1210-9.

37 Castro AS, Oliveira A, Lopes V. Pulmonary phaeohyphomycosis: a challenge to the clinician. Eur Respir Rev. 2013;22(128):187-8.

38 Nascimento JPM, López AMQ, Araújo MA, Araujo LA, Silva Filho EA. Airborne fungi in indoor hospital environments. Int J Curr Microbiol App Sci. $2019 ; 8(1): 2749-72$.

39 Silva DP, Lessa ILP, Medeiros MAS, Lacerda GAN, Mascarenhas MLVC, Costa ALC, et al. Fungal infections in preterm infants by yeasts of the genus Malassezia. Rev Enferm UFPE on line. 2018 Oct; 12(10):2836-43.

40 Colombo AL, Padovan ACB, Chaves GM. Current knowledge of Trichosporon spp. and trichosporonosis. Clin Microbiol Rev. 2011 Oct;24(4):682-700

41 Guého E, Smith MT, Hoog GS, Billon-Grand G, Christen R, Batenburg van der Vegte $\mathrm{WH}$. Contributions to a revision of the genus Trichosporon. Antonie Van Leeuwenhoek. 1992 May;61 (4):289-316.

42 Sugita T, Nakajima M, Ikeda R, Matsushima T, Shinoda T. Sequence analysis of the ribosomal DNA intergenic spacer 1 regions of Trichosporon species. J Clin Microbiol. 2002 May;40(50:1826-30.

43 Samonis G, Anatoliotaki M, Apostolakou H, Maraki S, Mavroudis D, Georgoulias V. Transient fungemia due to Rhodotorula rubra in a cancer patient: case report and review of the literature. Infection. 2001 Jun;29:173-6.

44 Alliot C, Desablens B, Garidi R, Tabuteau S. Opportunistic infection with Rhodotorula in cancer patients treated by chemotherapy: two case reports. Clin Oncol. 2000 Apr;12(2): $115-7$.

45 Huttova M, Kralinsky K, Horn J, Marinova I, lligova $\mathrm{K}$, Fric J, et al. Prospective study of nosocomial fungal meningitis in children-report of 10 cases. Scand J Infect Dis. 1998;30(5):485-7. 